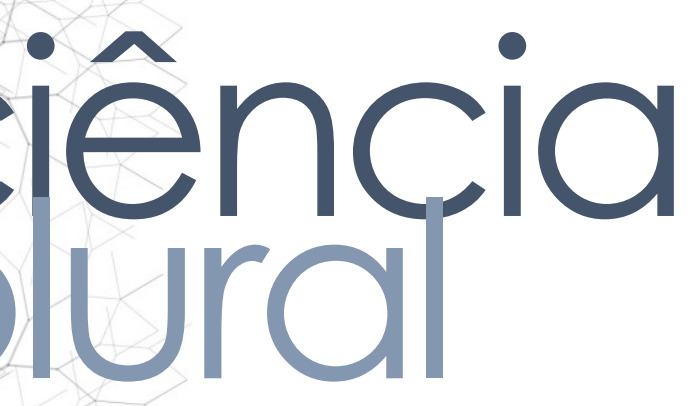

\title{
PERFIL DOS CASOS DE SÍFILIS CONGÊNITA NO RIO GRANDE DO NORTE: ESTUDO DE SÉRIE TEMPORAL
}

Profile of Congenital Syphilis cases in Rio Grande do Norte State: a Temporal Series Study

El perfil de los casos de sífilis congénita en Rio Grande do Norte: Estudio de Serie Temporal

Francisca Kelle de Sousa Ferreira • Enfermeira pela Universidade Federal de Campina Grande-UFCG • Pós-graduada pelo Programa de Residência Multiprofissional em Atenção Básica pela Universidade Federal do Rio Grande do Norte-UFRN • E-mail: keelleferreira@gmail.com

Ana Carine Arruda Rolim • Docente da UFRN • Doutora em Saúde Coletiva pela Faculdade de Ciências Médicas da Universidade Estadual de Campinas (FCM/Unicamp-SP) • E-mail: anacarine.rolim@gmail.com

Diego Bonfada • Docente da Universidade Federal do Rio Grande do Norte-UFRN - Doutor em Saúde Coletiva pela UFRN e pela Agència de Qualitat i Avaluació Sanitàries de Catalunya (AQuAS), Barcelona-ES (2015) • E-mail: diegobonfada@hotmail.com

Autora correspondente:

Francisca Kelle de Sousa Ferreira • E-mail: keelleferreira@gmail.com 


\section{ciênciála
pural}

\section{RESUMO}

Objetivo: Analisar a incidência, a mortalidade e o perfil dos casos de sífilis congênita no Rio Grande do Norte entre 2014 e 2018. Metodologia: Trata-se de um estudo epidemiológico, quantitativo, ecológico, realizado com dados de sífilis congênita registrados no Sinan. Resultados: A taxa de incidência e mortalidade por sífilis congênita duplicaram no Estado no período analisado. A maioria das crianças foi diagnosticada com menos de 7 dias após o nascimento, sendo mais predominante em filhos de mães com idade entre 20 e 29 anos, com baixa escolaridade e pardas, que foram diagnosticadas com sífilis recente e que tiveram acesso ao pré-natal. A maioria teve o diagnóstico da sífilis materna durante o pré-natal, mas não realizou tratamento adequado e o parceiro não recebeu tratamento. Conclusões: Os achados apontam para necessidade de estratégias para qualificação da atenção pré-natal com intuito de evitar a transmissão vertical, especialmente nesses grupos mais susceptíveis e vulneráveis.

Palavras-Chave: Sífilis Congênita. Transmissão vertical. Notificação de doenças. Cuidado pré-natal.

\section{ABSTRACT}

Objective: To analyze the incidence, mortality, and profile of cases of congenital syphilis at the Rio Grande do Norte between 2014 and 2018. Methodology: This is an epidemiological, quantitative, ecological study conducted with data on congenital syphilis registered at Sinan. Results: The incidence and mortality from congenital syphilis doubled in Rio Grande do Norte State during the analyzed period. Most children were diagnosed less than seven days after birth, being more prevalent in children of mothers aged 20 to 29 years, with low education and mixed-race, diagnosed with recent syphilis, and who had access to prenatal care. Most were diagnosed with maternal syphilis during prenatal care but did not receive adequate treatment, and the partner did not receive treatment. Conclusions: The findings show the need for strategies for quality prenatal care to avoid vertical transmission, especially in these most susceptible and vulnerable groups.

Keywords: Syphilis, Congenital. Infectious Disease Transmission, Vertical. Disease Notification. Prenatal Care.

\section{RESUMEN}

bjetivo: Analizar la incidencia, la mortalidad y el perfil de los casos de sífilis ongénita en el Río Grande del Norte entre 2014 y 2018. Metodología: Se trata de un studio epidemiológico, cuantitativo y ecológico realizado con los datos de sífilis ngénita registrados en el Sinan. Resultados: La incidencia y mortalidad por sífilis génita se duplicó en el estado de Río Grande do Norte durante el período alizado. La mayoría de los niños fueron diagnosticados en menos de siete días 


\section{ciência
piural}

después del nacimiento, siendo más prevalente en los hijos de madres de 20 a 29 años, con baja educación y mestizos, diagnosticados con sífilis reciente y que tuvieron acceso a la atención prenatal. La mayoría fueron diagnosticados de sífilis materna durante la atención prenatal pero no recibieron el tratamiento adecuado, y la pareja no recibió tratamiento. Conclusiones: Los hallazgos muestran la necesidad de estrategias de atención prenatal de calidad para evitar la transmisión vertical, especialmente en estos grupos más susceptibles y vulnerables.

Palabras clave: Sífilis Congénita. Transmisión Vertical de Enfermedad Infecciosa.

Notificación de Enfermedades. Atención Prenatal. 


\section{Introdução}

A sífilis congênita (SC) é considerada um problema de saúde pública e sua eliminação ou erradicação constitui um desafio para gestores, pesquisadores e profissionais de saúde. Pois, quando não diagnosticada e tratada adequadamente durante a gestação, pode provocar abortamento, má formação fetal e risco de recémnascido ou crianças apresentarem sinais clínicos preocupantes de SC1.

No Brasil, em 2018, foram registrados 26.219 casos de SC, com 241 óbitos infantis. De 2010 a 2018 constatou-se que a incidência da SC cresceu 3,8 vezes. O Rio Grande do Norte possui taxas de incidência de SC ainda maiores que a taxa nacional, o que denota possíveis falhas no pré-natal2,3.

Além disso, estudos destacam outros fatores de risco associados a incidência da SC, como baixa escolaridade, nível socioeconômico, múltiplos parceiros, parceiro não tratado e a falta do medicamento nas redes de atenção à saúde ${ }^{4,5,6}$. Ao refletir sobre esses preditores de risco, pode-se perceber que medidas eficientes e de baixo custo, feitas no espaço da Atenção Primária em Saúde (APS), podem ser importantes na prevenção do agravo7.

Nesse cenário, compreender a ocorrência e o perfil dos casos SC torna-se fundamental para fornecer subsídios na busca de medidas de prevenção, controle, redução da disseminação e interrupção da sua transmissibilidade, além de ter o potencial para formulação e fortalecimento de políticas públicas de combate à doença.

O presente estudo objetivou analisar a incidência, mortalidade e o perfil dos casos de sífilis congênita no Rio Grande do Norte entre 2014 e 2018.

\section{Metodologia}

Trata-se de um estudo epidemiológico, ecológico e de abordagem quantitativa, realizado a partir dos casos de SC no Rio Grande do Norte entre janeiro de 2014 a ezembro de 2018. Os dados foram coletados no Sistema de Informação de Agravos tificação (Sinan), obtidos através do Departamento de Informática do Sistema co de Saúde (Datasus), no período de julho a dezembro de 2019. 
As variáveis de interesse foram: número de casos, taxa de incidência e óbitos por SC em menores de um ano de idade; caracterização da sífilis congênita segundo faixa etária, escolaridade e cor da mãe; realização de pré-natal; momento do diagnóstico; esquema de tratamento; tratamento do parceiro, idade da criança no momento do diagnóstico e diagnóstico final.

As informações obtidas foram armazenadas em um banco de dados no Microsoft Excel, e posteriormente os dados foram organizados em tabelas e gráficos e analisados por meio de estatística descritiva e variância da incidência de SC ao longo do recorte temporal adotado. Utilizou-se dados secundários de domínio público, não havendo necessidade de autorização pelo comitê de ética em pesquisa.

\section{Resultados}

No Rio Grande do Norte, no período de 2014 a 2018, foram notificados 2.108 casos de SC no Sinan, sendo possível observar que no período analisado houve uma elevação substancial na ocorrência da doença, a taxa de incidência cresceu $111 \%$. A Figura 1 apresenta a incidência da SC por ano de diagnóstico.

Figura 1: Taxa de incidência de Sífilis Congênita por 1.000 nascidos vivos no Rio Grande do Norte entre os anos de 2014 e 2018. Caicó-RN, 2019.

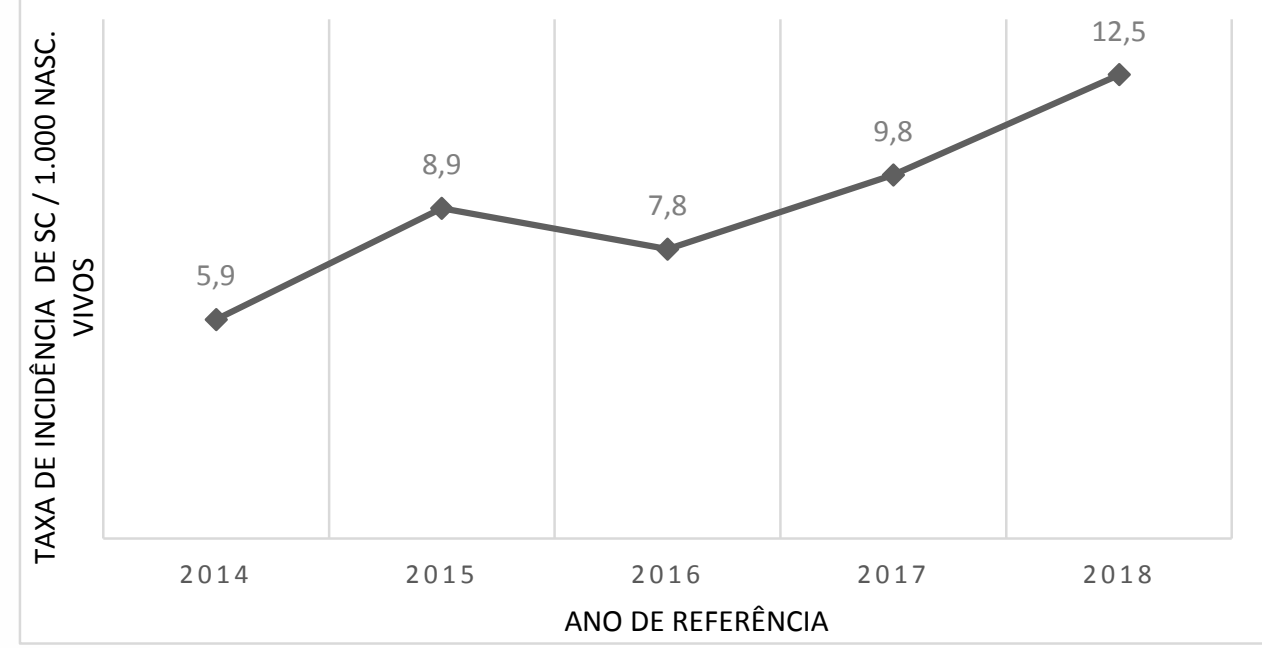

Fonte: Sinan/ Datasus

A Figura 2, apresenta a taxa de mortalidade por SC em menores de um ano (por .000 nascidos vivos) no Rio Grande do Norte, correspondente aos anos de 


\section{ciência
purala}

investigação. Verificou-se que no período estudado o número de óbitos por SC apresentou aumento de 157\%, com uma curva visivelmente crescente em 2016.

Figura 2: Coeficiente de mortalidade por sífilis congênita em menores de um ano, por 100.000 nascidos vivos, segundo ano do óbito no Rio Grande do Norte. Caicó-RN, 2019.

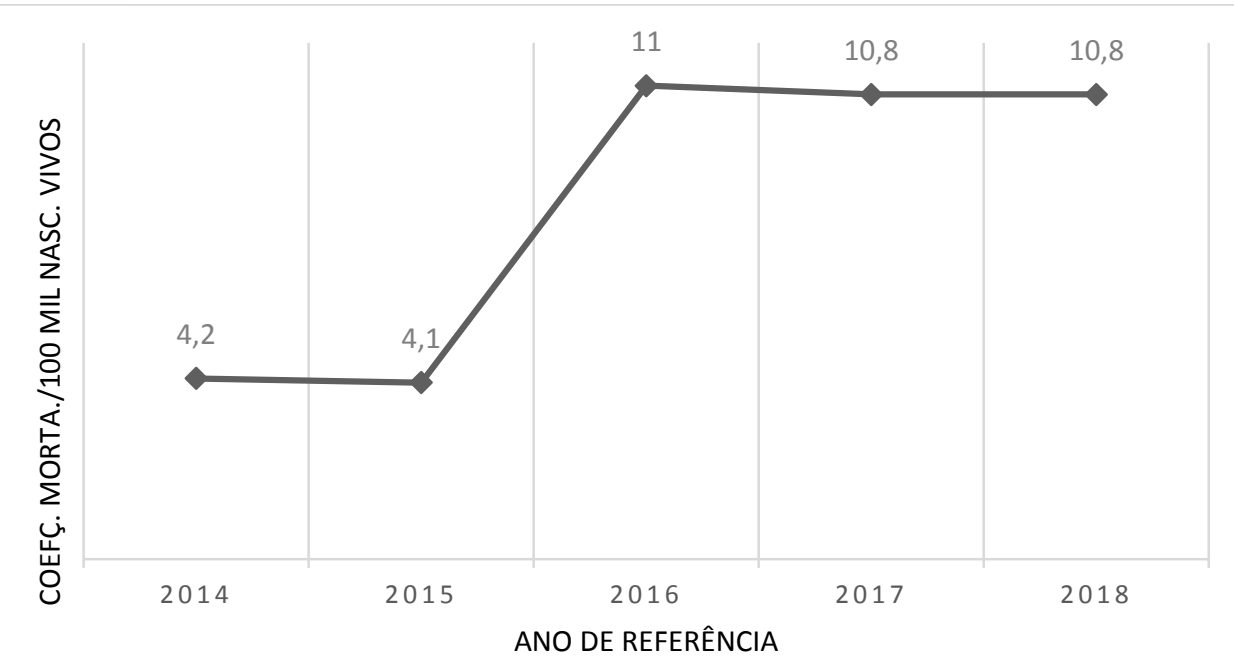

Fonte: Sinan/Datasus

Na Tabela 1, foram descritas as frequências absolutas e relativas das variáveis sociodemográficas dos casos notificados de SC. Em relação à variável idade no momento do diagnóstico, observou-se que quase a totalidade das crianças foram diagnosticadas com SC com menos de 7 dias após o nascimento.

Tabela 1: Caracterização sociodemográfica dos casos de sífilis congênita. Rio Grande do Norte, 2014 a 2018. $(\mathrm{n}=2.108)$. Caicó-RN, 2019.

\begin{tabular}{llcc}
\hline Variável & Categoria & $\mathbf{n}$ & $\mathbf{\%}$ \\
\hline Idade da criança no & Menos de 7 dias & 2.051 & 97,30 \\
momento do & 7 a 27 dias & 19 & 0,90 \\
diagnóstico & 28 a 364 dias & 31 & 1,47 \\
& 1 ano & 1 & 0,05 \\
& 2 a 4 anos & 2 & 0,09 \\
& 5 a 12 anos & 4 & 0,19 \\
& Total & $\mathbf{2 . 1 0 8}$ & $\mathbf{1 0 0}$ \\
\hline Faixa etária da mãe & 10 a 14 anos & 21 & 1,00 \\
& 15 a 19 anos & 494 & 23,43
\end{tabular}




\begin{tabular}{|c|c|c|c|}
\hline & 20 a 29 anos & 1.048 & 49,71 \\
\hline & 30 a 39 anos & 443 & 21,02 \\
\hline & 40 anos ou mais & 50 & 2,37 \\
\hline & Ignorado & 52 & 2,47 \\
\hline & Total & 2.108 & 100 \\
\hline \multirow{12}{*}{$\begin{array}{l}\text { Sífilis congênita } \\
\text { segundo escolaridade } \\
\text { da mãe }\end{array}$} & Analfabeto & 30 & 1,42 \\
\hline & $1^{\mathrm{a}}$ a $4^{\mathrm{a}}$ série incompleta & 164 & 7,78 \\
\hline & $4^{\text {a }}$ série completa & 93 & 4,41 \\
\hline & $5^{\mathrm{a}}$ a $8^{\mathrm{a}}$ série incompleta & 789 & 37,43 \\
\hline & Fundamental completo & 141 & 6,7 \\
\hline & Ensino médio & 297 & 14,09 \\
\hline & incompleto & 249 & 11,81 \\
\hline & Ensino médio completo & 18 & 0,85 \\
\hline & Superior incompleta & 08 & 0,38 \\
\hline & Superior completo & 319 & 15,13 \\
\hline & Não se aplica/Ignorado & 2.108 & 100 \\
\hline & Total & & \\
\hline \multirow[t]{7}{*}{ Raça ou cor da mãe } & Branca & 531 & 25,19 \\
\hline & Preta & 96 & 4,55 \\
\hline & Amarela & 06 & 0,3 \\
\hline & Parda & 1.318 & 62,52 \\
\hline & Indígena & 02 & 0,09 \\
\hline & Ignorada & 155 & 7,35 \\
\hline & Total & 2.108 & 100 \\
\hline
\end{tabular}

Fonte: SINAN/DATASUS

Quanto a variável idade materna, observa-se uma predominância na faixa etária entre 20 e 29 anos, e, em menor número, adolescentes. No que se refere a escolaridade das mães que tiveram filhos diagnosticados com sífilis congênita, a maior parte delas o concluíram o ensino fundamental e são de raça/cor parda. 


\section{ciência
piural}

Na Tabela 2, estão dispostas as variáveis relativas à assistência de pré-natal e parto, diagnóstico da sífilis e tratamento do parceiro dos casos de SC notificados.

Tabela 2: Aspectos relacionados a assistência de pré-natal e parto, diagnóstico da sífilis e tratamento do parceiro. Rio Grande do Norte, 2014 a 2018. Caicó-RN, 2019.

\begin{tabular}{|c|c|c|c|}
\hline Variável & Categoria & $\mathbf{N}$ & $\%$ \\
\hline \multirow[t]{5}{*}{ Diagnóstico final } & Sífilis congênita recente & 2.076 & 98,48 \\
\hline & Sífilis congênita tardia & 06 & 0,28 \\
\hline & Aborto por sífilis & 05 & 0,24 \\
\hline & Natimorto por sífilis & 21 & 1,00 \\
\hline & Total & 2.108 & 100 \\
\hline \multirow[t]{4}{*}{ Realização de pré-natal } & Sim & 1.778 & 84,35 \\
\hline & Não & 256 & 12,14 \\
\hline & Ignorado & 74 & 3,51 \\
\hline & Total & 2.108 & 100 \\
\hline \multirow{6}{*}{$\begin{array}{l}\text { Momento } \\
\text { diagnóstico }\end{array}$} & Durante o pré-natal & 1.085 & 51,47 \\
\hline & $\begin{array}{l}\text { No momento do parto/ } \\
\text { curetagem }\end{array}$ & 841 & 39,90 \\
\hline & Após o parto & 104 & 4,93 \\
\hline & Não realizado & 07 & 0,33 \\
\hline & Ignorado & 71 & 3,37 \\
\hline & Total & 2.108 & 100 \\
\hline \multirow{5}{*}{ Esquema de tratamento } & Adequado & 69 & 3,27 \\
\hline & Inadequado & 1.606 & 76,19 \\
\hline & Não realizado & 268 & 12,71 \\
\hline & Ignorado & 165 & 7,83 \\
\hline & Total & 2.108 & 100 \\
\hline \multirow{4}{*}{ Parceiro tratado } & Sim & 392 & 18,60 \\
\hline & Não & 1.263 & 59,91 \\
\hline & Ignorado & 453 & 21,49 \\
\hline & Total & 2.108 & 100 \\
\hline
\end{tabular}

te: Sinan/Datasus 
Observa-se que a maioria dos casos realizou acompanhamento na gestação e mais da metade receberam o diagnóstico da sífilis materna durante o pré-natal. Contudo, o tratamento materno da sífilis foi considerado inadequado ou não realizado e a maioria dos parceiros sexuais não foram tratados para sífilis.

\section{Discussão}

O Ministério da Saúde e Organização Pan-Americana da Saúde estabeleceram como meta para o ano de 2015 a eliminação da transmissão vertical da SC, sendo indicada a diminuição da taxa de incidência para menos de 0,5 por 1.000 nascidos vivos $^{8}$.

Entretanto, a incidência de SC no Rio Grande do Norte teve um comportamento crescente ao longo do período analisado. No ano de 2018, o estado apresentou incidência 25 vezes maior que preconizado, demonstrando que ainda se encontra distante de alcançar a eliminação da doença. Elevadas taxas de SC são encontradas também em diferentes regiões, todavia, o estado possui uma das maiores taxas de mortalidade infantil por SC do Brasil (10,8/100.000) $)^{2,9-11}$.

A elevação da incidência e de mortalidade por SC é algo preocupante que se configura como um problema de saúde pública no Rio Grande do Norte, revelando possíveis falhas nos serviços de saúde ofertados a população. Nesse cenário, torna-se fundamental que as políticas públicas e medidas de controle sejam fortalecidas na assistência pré-natal, via ampliação do acesso ao tratamento da sífilis e capacitação dos profissionais da APS sobre o manejo apropriado do agravo em uma lógica de atenção integral e em rede ${ }^{3}$.

O diagnóstico de sífilis no período neonatal precoce foi maior, resultados semelhantes foram encontrados em outros estados Brasileiros ${ }^{12,13}$. Na faixa etária de 20 a 29 anos houve um maior número de notificações de gestante com diagnóstico da sífilis, coincidindo com a idade em que ocorre também maior número de estações ${ }^{7,9,14,15}$.

Há ainda evidências de elevação de risco para sífilis entre mulheres pardas e baixa escolaridade, levantando a falta de informação enquanto hipótese plausível explicação do maior contágio entre elas $4,10,16$. Isso reforça a necessidade planejar 
ações, políticas de atenção e/ou programas de controle da sífilis, especialmente voltados a mulheres com esse perfil.

Houve elevada frequência do diagnóstico final classificado como SC recente, mesmo entre mães que tiveram acesso à assistência pré-natal. Alguns estudos que investigaram a realização do pré-natal e a ocorrência de SC no Brasil apresentaram resultados similares, demostrando que se trata de um agravo de importância para a saúde pública em âmbito nacional5,11,13,15,17.

É reconhecido que o acompanhamento pré-natal realizado de forma adequada constitui uma importante ferramenta para redução da incidência da SC7. Contudo, os dados expressam possíveis fragilidades, no diagnóstico oportuno e no manejo dos casos, revelando a necessidade de maior resolutividade dos serviços na APS e de fortalecimento da rede cegonha nas regionais de saúde $\mathrm{e}^{11,12}$.

Sobre o papel da APS, pode-se destacar dois elementos basilares, o diagnóstico e o tratamento para sífilis gestacional. O diagnóstico da infecção no pré-natal reflete importância de realizar a triagem sorológica oportunamente, com o devido suporte da rede diagnóstico na atenção secundária à saúde. Da mesma maneira, o tratamento adequado da sífilis no período gestacional caracteriza-se como uma importante medida para impedir a ocorrência da SC.

No entanto, este estudo revelou falhas no tratamento da infecção no Rio Grande do Norte, de maneira semelhante a Paraíba, estado vizinho, onde o tratamento para sífilis nas gestantes também foi considerado inadequado ou não realizado em $84 \%$ dos $\operatorname{casos}^{18}$.

Portanto, embora atualmente haja maior cobertura e adesão às consultas de prénatal e, consequentemente a realização de um diagnóstico mais precoce da sífilis, o tratamento das gestantes e seus parceiros ainda é predominantemente inadequado ${ }^{5,11}$.

Em pesquisa realizada em Alagoas, 63,66\% dos parceiros de gestantes com sífilis não foram tratados durante o pré-natal ${ }^{19}$, semelhante aos achados deste estudo. A não realização do tratamento pelo parceiro representa um entrave no controle e na revenção da SC, por ocasionar uma condição desencadeadora de reinfecção da stante $^{20}$. Dessa forma, torna-se importante lançar estratégias para proporcionar 
maior adesão da população masculina tanto durante a testagem como na adesão ao tratamento.

Uma visão arcaica sobre o papel do homem na sociedade reconhecidamente tem impactos sobre as políticas voltadas para saúde do homem e, nesse caso, também traz implicações para saúde da mulher e da criança. Diante disso, é importante o uso de ferramentas como a educação popular em saúde feita em grupo ou individualmente, como instrumento de reconstrução da identidade masculina para questões sensíveis à saúde coletiva.

Este estudo apresenta como fortaleza, o uso de dados secundários que apresentam como vantagens, fácil acesso e baixo custo para a coleta das informações. Em relação as limitações na execução do estudo, apontam-se a impossibilidade em mensurar subnotificações e preenchimento incompleto das fichas de notificações, evidenciado por algumas informações ignoradas.

Os resultados apresentados reforçam a necessidade de medidas e estratégias para prevenção e controle da SC, especialmente na qualificação do pré-natal. Nesse sentido, evidencia-se a importância da garantia da obtenção do resultado do VDRL em tempo oportuno pelas gestantes e seus parceiros, com o fortalecimento da rede de apoio diagnóstico.

Ressalta-se também que os resultados desta pesquisa podem contribuir para formulação de políticas e estratégias relacionadas à prevenção e ao controle da sífilis, que propiciem não só diagnóstico, mas também adesão ao tratamento, especialmente entre a populações consideradas socialmente mais vulneráveis a infecção.

\section{Conclusões}

O perfil dos casos de SC encontrado neste estudo demostra que a incidência e a taxa de mortalidade por SC no Rio Grande do Norte, apresenta comportamento crescente durante o período analisado. A taxa de incidência cresceu $111 \%$ e o número de óbitos por SC apresentou aumento de $157 \%$.

Quanto a caracterização sociodemográfica dos casos de SC, o diagnóstico da fecção foi predominante na faixa etária de até seis dias do nascimento, houve maior 
ocorrência de SC em filhos de mães com idade entre 20 a 29 anos, com baixa escolaridade e pardas.

Embora a maioria das gestantes tenha tido acesso à atenção pré-natal, os achados demostram fragilidades na assistência pré-natal, apresentando baixa efetividade das ações de prevenção, esquema de tratamento inadequado de gestantes e o não tratamento do parceiro, essas falhas contribuem para o aumento da incidência de SC.

\section{Referências}

1. Peres MLAHS, Ferreira WFS, Oliveira EM. Sífilis congênita: uma problemática em saúde pública. Rev Univ Vale do Rio Verde [Internet]. 2019 [citado 2020 Jan 6];17(1):1-11. Disponível em:

http://periodicos.unincor.br/index.php/revistaunincor/article/view/4636/pdf_89 5. http://dx.doi.org/10.5892/ruvrd.v17i1.4636.g10951660

2. Ministério da Saúde (BR). Secretaria de Vigilância em Saúde. Departamento de DST, AIDS e Hepatites Virais. Boletim Epidemiológico de sífilis. Brasília: Ministério da Saúde, 2019.

3. Araújo E C, Monte P C B, Haber A N C A. Avaliação do pré-natal quanto à detecção de sífilis e HIV em gestantes atendidas em uma área rural do estado do Pará, Brasi. Rev Pan amaz de Saúde [Internet]. 2018 [citado em 2019 Nov 30];9(1):3339. Disponível em:

http:/ / revista.iec.gov.br/submit/index.php/rpas/article/view/349/232.

http:/ / dx.doi.org/10.5123/s2176-62232018000100005

4. Souza BSO, Rodrigues RM, Gomes RML. Análise epidemiológica de casos notificados de sífilis. Rev Soc Bras Clin Med [Internet]. 2018 [citado 2019 Nov 25];16(2):94-98. Disponível em:

http:/ / docs.bvsalud.org/biblioref/2018/09/913366/16294-98.pdf

5. Zoilo CS, Barbosa EPM, Barbosa JA, Paes LBO. Fatores maternos associados à transmissão vertical da sífilis congênita. Cuidarte Enfermagem [Internet]. 2018 [citado em 2019 Nov 20];12(2):211-217. Disponível em:

http://www.webfipa.net/facfipa/ner/sumarios/cuidarte/2018v2/211_217.pdf

Tavares LT, Albergaria TFS, Guimarães MAP, Pedreira RBS, Pinto Junior EP. ortalidade infantil por causas evitáveis na Bahia 2000-2012. Rev Eletron Comun Inf ov Saúde [Internet]. 2016 [citado 2020 Jan 26];10(3):1-10. Disponível em: tps:/ / www.reciis.icict.fiocruz.br/index.php/reciis/article/view/1044. Doi: 29397/ reciis.v10i3.1044. DOI: https:/ / doi.org/10.29397/ reciis.v10i3.1044 


\section{ciêncíncia
puúl}

7. Ferreira AG, Gomes LA, Medeiros RCT, Leonidas RAM, Medeiros JP. Perfil dos Casos de Sífilis Congênita no Município de Natal / RN no Período de 2007 a 2015. Rev Saúde em foco [Internet]. 2018 [citado em 2020 Jan 6];5(1):4-27. Disponível em: http:/ / www4.fsanet.com.br/revista/index.php/saudeemfoco/article/view/1651. http:/ / dx.doi.org/10.12819/rsf.2018.5.1.1

8. Ministério da Saúde (BR). Transmissão vertical do HIV e sífilis: estratégias para redução e eliminação. Brasília: Ministério da Saúde, 2014. 18p

9. Lima VC, Mororó RM, Martins MA, Ribeiro SM, Linhares MSC. Perfil epidemiológico dos casos de sífilis congênita em um município de médio porte no nordeste brasileiro. J. Heath Biol Sci [Internet]. 2017 [citado 2020 Jan 24];1(5):56-61. Disponível em: https:/ / periodicos.unichristus.edu.br/jhbs/article/view/1012/ 406. Doi:10.12662/2317-3076jhbs.v5i1.1012.p.56-61.2017

10. Cavalcante PAM, Pereira RBL, Castro JGD. Sífilis gestacional e congênita em Palmas, Tocantins, 2007-2014. Epidemiol. Serv. Saúde [Internet]. 2017 [citado 2020 Jan 2];26(2):265-274. Disponível em: http:/ / www.scielo.br/scielo.php?pid=S223796222017000200255\&script=sci_abstract\&tlng=pt. https://doi.org/10.5123/s167949742017000200003.

11. Moreira KFA, Oliveira DM, Alencar LN, Cavalcante DFB, Pinheiro AS, Orfão NH. Perfil dos casos notificados de sífilis congênita. Cogitare Enfermagem [Internet]. 2017 [citado 2020 Jan 19];22(2) Disponível em: https:/ / www.redalyc.org/jatsRepo/4836/483654815007/html/index.html. Doi: $10.5380 /$ ce.v22i1.48949

12. Amâncio V C, Graciano A R, Cozer A M, Assis L P F, Dias D C S. Epidemiologia da sífilis congênita no estado de Goiás. Rev. Educ. Saúde [Internet]. 2016 [citado 2019 Nov 30];4(2):58-63. Disponível em:

http:// periodicos.unievangelica.edu.br/index.php/educacaoemsaude/article/view $/ 2017$

13. Oliveira JS, Santos JV. Perfil epidemiológico da sífilis congênita no estado da Bahia, no período de 2010 a 2013. Rev. Eletrôn. Atualiza Saúde [Internet]. 2015 [citado 2019 Dez 19];2(2):20-30. Disponível em: http:/ / atualizarevista.com.br/article/ perfil-epidemiologico-da-sifilis-congenita-noestado-da-bahia-no-periodo-de-2010-a-2013-v-2-n-2/ .

Azevedo DMS, Reis RBS, Teles MF. Incidência e caracterização dos casos de sífilis ngênita na maternidade de um hospital do sudoeste Baiano. Rev. Mult. Psic ternet]. 2019 [citado 2019 Dez 3];13(43):387-397. Disponível em: ps://idonline.emnuvens.com.br/id/article/view/1542 
15. Benito LAO, Souza WN. Perfil epidemiológico da sífilis congênita no Brasil no período de 2008 a 2014. Univ: Ciências da Saúde [Internet]. 2016 [citado 2019 Dez 2];14(2):97-104. Disponível em:

https:/ / www.researchgate.net/publication/311938866_Perfil_epidemiologico_da_si filis_congenita_no_Brasil_no_periodo_de_2008_a_2014 DOI:

http:/ / dx.doi.org/10.5102/ucs.v14i2.3811

16. Domingues RMSM, Leal MC. Incidência de sífilis congênita e fatores associados à transmissão vertical da sífilis: dados do estudo Nascer no Brasil. Cad. Saúde pública [Internet]. 2016 [citado 2020 Jan 8];32(6):1-12. Disponível em:

https:/ / www.scielo.br/scielo.php?pid=S0102-

311X2016000605002\&script=sci_abstract\&tlng=pt. http://dx.doi.org/10.1590/0102$311 \times 00082415$.

17. Guimarães TA, Alencar LCR, Fonseca LMB, Gonçalves MMC, Silva MP. Sífilis em gestantes e sífilis congênita no Maranhão. Arq. Ciênc. Saúde [Internet]. 2018 [citado 2020 Jan 22];25(2):24-30. Disponível em:

http:/ / www.cienciasdasaude.famerp.br/index.php/racs/article/view/1023/759. DOI: https:/ / doi.org/10.17696/2318-3691.25.2.2018.1023

18. Alcântara TT, Alcântara IT, Guerreiro JV, Nascimento Neto JM. Perfil epidemiológico da sífilis congênita no estado da Paraíba, 2007 a 2016. Brazilian Journal of Surgery and Clinical Research - BJSCR [Internet]. 2017 [citado 2019 Dez 26];18(3):21-26. Disponível em:

https:/ / pdfs.semanticscholar.org/ac7a/8e02666acf91422beac4d8dbba3e0f68a489.pdf

19. Leite JKS, Medeiros CDP, Leite BSS, Cavalcante JBN. Perfil epidemiológico e sócio demográfico da sífilis congênita no estado de Alagoas durante o período de 2010 a 2017. Gep news [Internet]. 2019 [citado 2020 Jan 29];2(2):176-181. Disponível em: http:/ / www.seer.ufal.br/index.php/gepnews/article/view/7897

20. Vasconcelos MIO, Oliveira KMC, Magalhães AHR, et al. Sífilis na gestação: estratégias e desafios dos enfermeiros da atenção básica para o tratamento simultâneo do casal. Rev Bras Promoç Saúde [Internet]. 2016 [citado 2020 Jan 8]; 29:85-92. Disponível em: https:// periodicos.unifor.br/RBPS/article/view/6409. DOI: http:/ /dx.doi.org/10.5020/18061230.2016.sup.p85 